\title{
Evidenzbasierte Informationen auf Deutsch
}

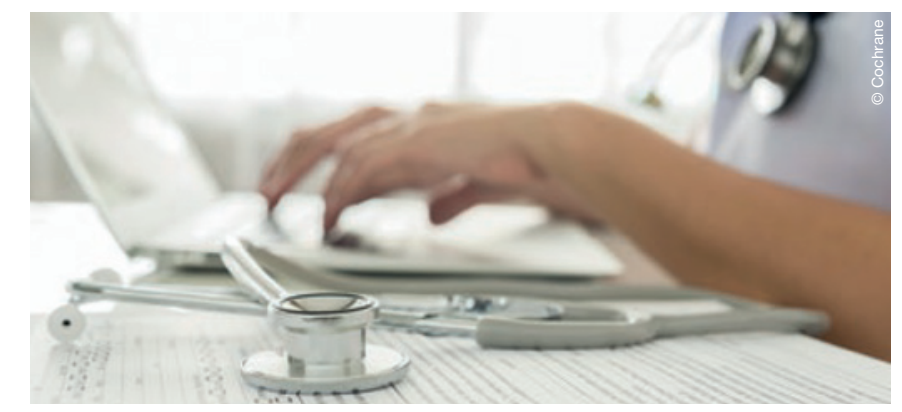

\author{
Wir alle treffen ständig Entscheidungen, die mit unserer Gesundheit \\ zu tun haben. Cochrane Österreich hilft dabei, verlässliche Informationen \\ für solche Entscheidungen in deutscher Sprache zu liefern.
}
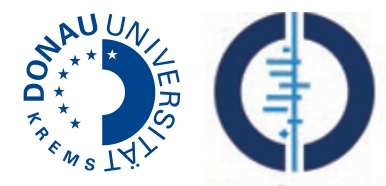

Cochrane Österreich

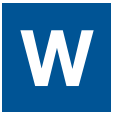
ie wirksam sind Reisebeschränkungen zur Eindämmung der Coronavirus-Erkrankung (COVID-19)? Wie zuverlässig sind Corona-Antigen-Schnelltests? Zu solchen Fragen suchen Forscherteams des internationalen Cochrane Netzwerkes die beste verfügbare Evidenz aus wissenschaftlichen Studien und fassen diese in Form von Cochrane Reviews zusammen. Diese Übersichtsarbeiten schaffen eine ausgewogene Grundlage für gesundheitsrelevante Entscheidungen. Das systematische Aufarbeiten von Evidenz war aber schon vor der Corona-Pandemie wichtig.

Cochrane setzt sich seit mehr als 25 Jahren dafür ein, dass Entscheidungen in der Gesundheitsversorgung evidenzbasiert sind. Aktuell stellt das unabhängige Forschungsnetzwerk mehr als 7500 Cochrane Reviews zu unterschiedlichen Gesundheitsfragen bereit. Einziges Manko - Cochrane Reviews werden auf Englisch publiziert. Das hält viele Personen im deutschen Sprachraum davon ab, Cochrane Evidenz für ihre Entscheidungsfindung zu nutzen.

\section{Cochrane bloggt auf Deutsch}

Wir - das Team von Cochrane Österreich - setzen uns dafür ein, dass Evidenz in deutscher Sprache verfügbar ist. Gemeinsam mit Cochrane Deutschland und Cochrane Schweiz betreiben wir den deutschsprachigen Blog www.wissenwaswirkt.org. Dort schreiben wir über die neuesten Erkenntnisse aus Cochrane Reviews. Aktuell läuft eine Blog-Serie zum Thema „Gesundes Altern“. Wir erstellen auch kurze Videos, die Methoden der evidenzbasierten Medizin einfach erklären. Unser Blog richtet sich an Angehörige aller Gesundheitsberufe und Menschen, die sich über verschiedene Gesundheitsthemen und evidenzbasierte Methoden informieren wollen. Zusätzlich übersetzen wir Zusammenfassungen von Cochrane Reviews. Mittlerweile sind bereits mehr als 2000 Übersetzungen verfügbar: www.cochrane.org/de/evidence.

\section{Evidenz für die medizinische und pflegerische Praxis}

Cochrane Österreich betreibt auch Anfrage-Services für individuelle Fragen, die sich in der Versorgung von Patientinnen und Patienten ergeben. Das evidenzbasierte Informationszentrum für Ärztinnen und Ärzte beantwortet Fragen aus der medizinischen Praxis (www.ebm-info.at); das evidenzbasierte Informationszentrum für Pflegende bearbeitet pflegerelevante Fragen (www.ebninfo.at).

\section{Gesundheitsmythen im Fakten-Check}

Für die allgemeine Bevölkerung in Österreich betreiben wir schon seit vielen Jahren die Fakten-Check Plattform Medizin-Transparent.at. Das Team von Medizin-Transparent.at überprüft die wissenschaftlichen Belege für Gesundheitsbehauptungen und veröffentlicht evidenzbasierte Informationen in leicht verständlicher Sprache. Auch hier liegt der Schwerpunkt zurzeit auf COVID-19: Mehr als 30 Artikel befassen sich bereits mit Corona-Mythen. Ganz aktuell wurden Informationen rund um die Corona-Impfungen veröffentlicht (www.medizin-transparent.at).

Kontakt: Dr. Barbara Nußbaumer-Streit, MSc BSc, Co-Direktorin Cochrane Österreich,

Donau-Universität Krems, mehr Infos und Newsletter: www.cochrane.at Cochrane Österreich wird gefördert vom Niederösterreichischen Gesundheits- und Sozialfonds. 